\title{
Geranylgeranyl Transferase
}

National Cancer Institute

\section{Source}

National Cancer Institute. Geranylgeranyl Transferase. NCI Thesaurus. Code C41056.

Alpha-beta heterodimeric human GGT ase-I transfers a geranylgeranyl moiety to the Cys of a C-terminal CAAX motif in candidate proteins as a means of membrane localization. The conserved 48-kDa alpha subunit of GGT ase-I is identical to the alpha subunit (FNTA) of Farnesyl Protein Transferase. The conserved 42-kDa Protein Geranylgeranyltransferase Type I Beta Subunit (PGGT 1B Gene), containing 4 PFT B repeats, is distinct from the beta subunit (FNTB) of Farnesyl Protein Transferase; FNT B and PGGT 1B are catalytic for specific substrates. The mammalian counterpart of CDC43 (S. cerevisiae) and responsible for peptide binding, PGGT1B acts on Rac1, Rac2, Rap1A, and Rap1B. Interacting with T GF-beta and Activin Type I receptors, FNTA is likely a key component of p21ras signaling, a farnesyltransferase substrate. ( $\mathrm{NCl})$ 